\title{
Chitosan immunomodulatory properties: perspectives on the impact of structural properties and dosage
}

\author{
David Fong ${ }^{1}$ \& Caroline D Hoemann ${ }^{*}, 1,2,3$ \\ ${ }^{1}$ Institute of Biomedical Engineering, École Polytechnique, Montreal, QC, H3T 1J4, Canada \\ ${ }^{2}$ Department of Chemical Engineering, École Polytechnique, Montreal, QC, H3T 1J4, Canada \\ ${ }^{3}$ Bioengineering Department, George Mason University, Institute for Advanced Biomedical Research, Manassas, VA 20110, USA \\ * Author for correspondence: Tel.: +1 703993 5884; Fax: +1 703993 2077; choemann@gmu.edu
}

'The precise difficulty in saying 'chitosan is pro-inflammatory' is that 'inflammation' covers a very broad range of cellular and molecular responses that in fact can be beneficial or harmful depending on the type and degree of inflammation."

First draft submitted: 26 May 2017; Accepted for publication: 30 May 2017; Published online: 14 September 2017

Keywords:biomaterials • chitin • chitosan • inflammation • interferon • interleukin-1 beta • interleukin-1 receptor antagonist • macrophage

Due to its abundance, biodegradability and cytocompatibility, the polysaccharide chitosan has been investigated for use in a wide range of biomedical applications, including tissue engineering scaffolds [1,2], vectors for drug and gene delivery [3] and vaccine adjuvants [4]. Interestingly, chitosan exhibits considerable immunostimulatory activity, by inducing innate immune cells to release a wide range of pro- and anti-inflammatory cytokines, chemokines, growth factors and bioactive lipids [5]. Release of these mediators can have a significant impact on the performance of the different biomedical devices where chitosan is used. Despite considerable advances in our understanding of chitosan-innate immune responses in vitro, challenges persist in predicting whether a chitosan-based medical device will elicit pro- or anti-inflammatory responses in vivo. Addressing this knowledge gap is critical for a better and safer translation use of chitosan in biomedical devices. This paper will discuss important and recent advances in chitosan biology, and perspectives on the next unanswered questions.

Because chitosan is derived by chemical modification of a naturally sourced polymer, chitin, it is important to note that chitosan does not represent a single polysaccharide. Chitosan encompasses a family of linear polymers containing $\beta-\mathrm{O}-(1-4)$-linked glucosamine (GlcN) and variable levels of $\mathrm{N}$-acetyl glucosamine (GlcNAc). Deacetylation of chitin using enzymatic or chemical hydrolysis produces chitosan chains with anywhere from 40 to $100 \% \mathrm{GlcN}$ content, also known as the degree of deacetylation (DDA) [3]. Following chemical deacetylation, GlcNAc residues are distributed in clusters (block acetylation); however, chitosans with homogenously arranged GlcNAc residues (random acetylation) can also be obtained by preparing a fully deacetylated chitosan that is then reacetylated to a particular DDA level [6]. In addition to the DDA and acetylation pattern, molecular weight (MW) is another important structural property that strongly influences chitosan biological and physicochemical properties [3]. Parent chitosan chains typically range from 200,000 to 1,000,000 weight-average MW; however, smaller average MW, even very small oligomers, can be targeted with enzymes or different acids that can specifically hydrolyze the $\beta-\mathrm{O}-(1-4)$-linkages without substantially changing the DDA level [3,7-9]. The important point here is that there are a variety of possible approaches to produce chitosan, using chitin or chitosan from many different sources and suppliers. Many researchers have investigated chitosans with distinct DDA, MW, polydispersity, purity, form and dose, all of which can impact biological-innate immune responses. This has ultimately led to some confusion and divergent opinion as to whether chitosan is a pro-inflammatory or anti-inflammatory biomaterial.

Although immune-modulating properties of chitosan have been studied for over 30 years [10], intracellular chitosan response pathways have only been recently begun to be elucidated. Currently, the best-described intracellular signaling pathways involve cGAS-STING, and NLRP3 $[4,11,12]$. These pathways were shown to be deployed follow- 


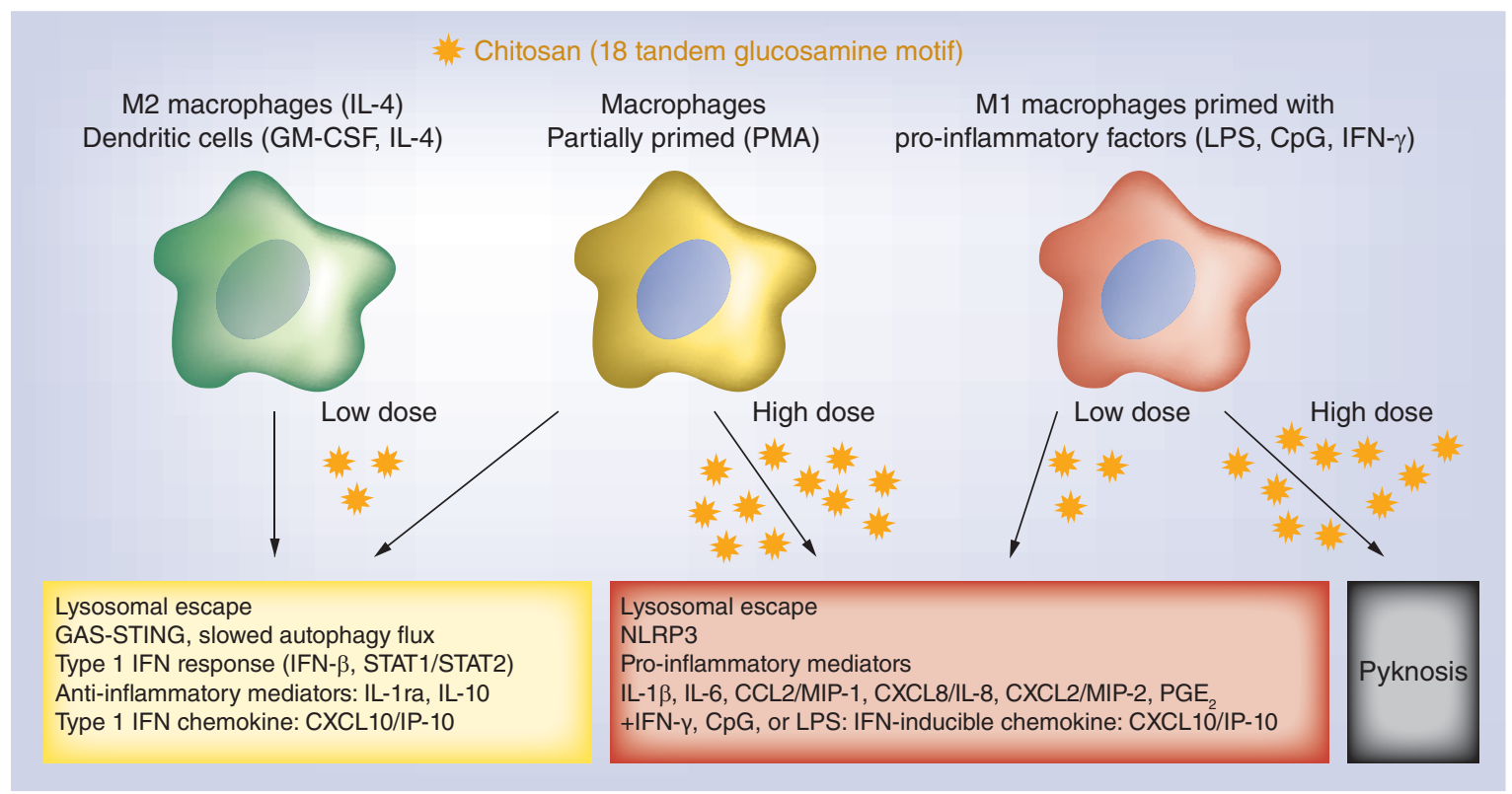

Figure 1. Inflammatory responses to chitosan depend on prior immune cell activation state, chitosan dose, and the presence of a tandem glucosamine motif. In macrophages primed with phorbol ester (PMA), low doses of chitosan induce type 1 IFN, leading to increased IL-1ra and CXCL10/IP-10 release and low levels of IL-1 $\beta$ and PGE 2 . High doses of chitosan activate the inflammasome, which leads to increased release of IL-1 $\beta$ and PGE 2 , and suppressed release of IL-1ra and CXCL10/IP-10. When macrophages are polarized towards an M1 state, chitosan enhances the release of pro-inflammatory cytokines. In macrophages polarized towards an M2 state, chitosan enhances the release of anti-inflammatory cytokines.

ing macrophage exposure to chitosans with 80 to $98 \%$ DDA and 3 to $400 \mathrm{kDa}$ in MW. The cGAS-STING pathway triggers a type 1 IFN response and specific downstream expression of the chemokine CXCL10/IP-10 [4,12]. Additionally, type 1 IFN responses, which signal through STAT1/STAT2 activation, induce the release of a well-known and therapeutically relevant anti-inflammatory factor, IL-1 ra [12]. By contrast, activation of NLRP3 leads directly to inflammasome activation and to the release of pro-inflammatory factors IL-1 $\beta$ and PGE $2[11,12]$. These two types of cytokine responses have been reported in both primary and cell line-derived human and mouse macrophage models as well as in vivo, providing great confidence that these responses to chitosan are reproducible and conserved across different species.

Most remarkably, these two signaling complexes were recently linked in the context of macrophage infection by intracellular pathogens such as Mycobacterium tuberculosis. In early intracellular pathogen infection, macrophages produce low levels of type 1 IFN, which in a paracrine manner induces the release of IL-1ra and IL-10 [13]. IL-10 suppresses the production of pro-inflammatory cytokines whereas IL-1 ra antagonizes IL-1 $\beta$ autocrine signaling. This promotes host cell and thus pathogen survival by deceiving host immunity to downregulate pro-inflammatory signals that could result in cell death by pyknosis [13]. High multiplicity of infection can trigger strong inflammasome activation and high levels of $\mathrm{PGE}_{2}$. In this case, high levels of $\mathrm{PGE}_{2}$ suppress type 1 IFN, IL-1ra and IL-10 production [13]. Recently, we reported that a selected number of chitosans (3-10 kDa, 98\% DDA; 10 and $190 \mathrm{kDa} ; 80 \%$ DDA, block-acetylated) in a particulate form mimic this model of macrophage infection by intracellular pathogens [12]. At low doses, chitosans containing the motif with a minimum $3000 \mathrm{Da}$ consecutive GlcN residues ( $\sim 18$ tandem GlcNs) induced a potent type 1 IFN response in macrophages without activating the inflammasome [12]. At higher doses, the same chitosans activated the inflammasome without eliciting a type 1 IFN response [12]. These interesting findings suggest that macrophages use the same sensing mechanisms to produce anti-inflammatory signals after chitosan particle or intracellular pathogen uptake. Lysosomal escape was found to be the novel and common mimetic event (Figure 1) [12]. While it has been reported that chitosan intrinsic properties play a significant role on the release of pro- or anti-inflammatory signals [14-16], the impact of dose was only recently recognized. This newly identified effect of dose highlights the potential to fine-tune chitosan use, not 
only in terms of structural properties, but also dose, to elicit or avoid specific immunomodulatory responses for a desired biomedical application.

When interpreting findings from the literature, there are many variables related to the experimental design that may drastically influence whether specific cytokine responses to chitosan stimulation are observed. These include properties of the chitosan preparation (i.e., DDA, MW, polydispersity), chitosan dosage, the cellular model used (e.g., primary or cell line, murine or human origin) and the presence of pro-inflammatory costimulation agents (e.g., LPS, CpG or IFN- $\gamma$ ) $[4,5]$. For instance, IL-1 $\beta$ production in macrophages following chitosan stimulation is routinely observed in macrophages primed with CpG or LPS [16-18], provided that the chitosan contains at least $3000 \mathrm{Da}$ consecutive GlcN monomers, and moderate chitosan doses are used $\left(\sim 50 \mu \mathrm{g} \cdot \mathrm{ml}^{-1}\right)$ [12]. When macrophages are treated with very high doses of chitosan, cell pyknosis is observed (Figure 1) [19]. These sources of variability emphasize why it is crucial to fully characterize the chitosan intended for use in biological studies. Far too often, the chitosan used have either been partially characterized for DDA or MW (i.e., DDA determined to be above or below a certain value, or MW between certain range with no average value or polydispersity index), or characterized thoroughly for only one of the two properties, leaving the other properties unknown [5]. Endotoxin content, which is rarely reported in the chitosan preparation, can also confound data interpretation.

The critical effect of chitosan dose in vitro on differential cytokine induction by macrophages (i.e., induction of a type 1 IFN response vs activation of the inflammasome) begs the question of what happens in vivo and more specifically how chitosan dose influences immune cell cytokine production in vivo. There are multiple attributes from the in vivo setting that the in vitro scenario cannot reproduce. For one, macrophage, neutrophil, mast cell and dendritic cell monoculture models do not consider the involvement of other immune cells. Yet, it is well known that a variety of innate immune cell types are elicited by chitosan, interact among each other and contribute in different ways to the host response to chitosan. Next, molecules in the extracellular environment and cell-to-cell interactions are largely simplified in vitro, and it is likely that this can influence immune cell response to chitosan. For instance, chitosan can interact with serum proteins, and this significantly affects how chitosan is recognized and presented to cells, which can potentially have an important impact on host response to chitosan [20]. Measuring the effects of chitosan dose and structure in an in vivo setting will be important to pursue and will provide valuable insights on host responses to chitosan.

Chitosan is the only natural cationic polysaccharide and has easily tunable properties that make it an attractive material for biomedical applications. Its immunomodulatory effects are rather wide, as chitosan can induce, based on structural properties and dosage, a plethora of cytokines of pro- or anti-inflammatory nature. Whether these pro- or anti-inflammatory responses are good or bad ultimately depends on the context. The precise difficulty in saying 'chitosan is pro-inflammatory' is that 'inflammation' covers a very broad range of cellular and molecular responses that in fact can be beneficial or harmful depending on the type and degree of inflammation. Inflammatory cell infiltration is necessary and beneficial for wound repair, but excessive cell infiltrate accompanied by classical immune cell activation can result in tissue damage. Inflammatory responses to chitosan must be evaluated with the perspective of how cellular, molecular and tissue responses are expected to influence the safety and performance of the biomedical device. Structure-function models are being established to predict both release of pro- or antiinflammatory cytokines from immune cells in vitro. Developing relevant models in vivo will be critical to designing biomaterials and devices containing chitosan that capitalize or minimize specific inflammatory responses.

\section{Financial \& competing interests disclosure}

This work was funded by the Canadian Institute of Health Research (MOP 303615-BME). CD Hoemann is a shareholder and on the Board of Directors of Ortho RTi. The authors have no other relevant affiliations or financial involvement with any organization or entity with a financial interest in or financial conflict with the subject matter or materials discussed in the manuscript apart from those disclosed.

No writing assistance was utilized in the production of this manuscript.

\section{Open access}

This work is licensed under the Creative Commons Attribution 4.0 License. To view a copy of this license, visit http:// creativecommons.org/licenses/by/4.0/ 


\section{References}

1 Stanish WD, Mccormack R, Forriol F et al. Novel scaffold-based BST-CarGel treatment results in superior cartilage repair compared with microfracture in a randomized controlled trial. J. Bone Joint Surg. Am. 95(18), 1640-1650 (2013).

2 Hoemann CD, Hurtig M, Rossomacha E et al. Chitosan-glycerol phosphate/blood implants improve hyaline cartilage repair in ovine microfracture defects. J. Bone Joint Surg. Am. 87(12), 2671-2686 (2005).

3 Buschmann MD, Merzouki A, Lavertu M, Thibault M, Jean M, Darras V. Chitosans for delivery of nucleic acids. Adv. Drug Del. Rev. 65(9), 1234-1270 (2013).

4 Carroll EC, Jin L, Mori A et al. The vaccine adjuvant chitosan promotes cellular immunity via DNA sensor cGAS-STING-dependent induction of type I interferons. Immunity 44(3), 597-608 (2016).

5 Hoemann CD, Fong D. Immunological properties of chitosan for biomedical applications. In: Chitosan Based Biomaterials Volume 1: Fundamentals. Jennings JA, Burngardner JD (Eds). 45-76 (2017).

6 Varum KM, Anthonsen MW, Grasdalen H, Smidsrod O. High-field NMR-spectroscopy of partially N-deacetylated chitins (chitosans).1. Determination of the degree of $\mathrm{N}$-acetylation and the distribution of $\mathrm{N}$-acetyl groups in partially $\mathrm{N}$-deacetylated chitins (chitosans) by high-field NMR-spectroscopy. Carbohydr. Res. 211(1), 17-23 (1991).

7 Lavertu M, Methot S, Tran-Khanh N, Buschmann MD. High efficiency gene transfer using chitosan/DNA nanoparticles with specific combinations of molecular weight and degree of deacetylation. Biomaterials 27(27), 4815-4824 (2006).

8 Tommeraas K, Varum KM, Christensen BE, Smidsrod O. Preparation and characterisation of oligosaccharides produced by nitrous acid depolymerisation of chitosans. Carbohydr. Res. 333(2), 137-144 (2001).

9 Rinaudo M, Pavlov G, Desbrieres J. Influence of acetic acid concentration on the solubilization of chitosan. Polymer 40(25), 7029-7032 (1999).

10 Nishimura K, Nishimura S, Nishi N, Saiki I, Tokura S, Azuma I. Immunological activity of chitin and its derivatives. Vaccine 2(1), 93-99 (1984).

11 Bueter CL, Lee CK, Wang JP, Ostroff GR, Specht CA, Levitz SM. Spectrum and mechanisms of inflammasome activation by chitosan. J. Immunol. 192(12), 5943-5951 (2014).

12 Fong D, Gregoire-Gelinas P, Cheng AP et al. Lysosomal rupture induced by structurally distinct chitosans either promotes a type 1 IFN response or activates the inflammasome in macrophages. Biomaterials 129, 127-138 (2017).

13 McNab F, Mayer-Barber K, Sher A, Wack A, O’Garra A. Type I interferons in infectious disease. Nat. Rev. Immunol. 15(2), 87-103 (2015).

14 Vasconcelos DP, Fonseca AC, Costa M et al. Macrophage polarization following chitosan implantation. Biomaterials 34(38), 9952-9959 (2013).

15 Gudmundsdottir S, Lieder R, Sigurjonsson OE, Petersen PH. Chitosan leads to downregulation of YKL-40 and inflammasome activation in human macrophages. J. Biomed. Mater. Res. A 103(8), 2778-2785 (2015).

16 Fong D, Ariganello MB, Girard-Lauziere J, Hoemann CD. Biodegradable chitosan microparticles induce delayed STAT-1 activation and lead to distinct cytokine responses in differentially polarized human macrophages in vitro. Acta Biomater. 12, 183-194 (2015).

17 Bueter CL, Lee CK, Rathinam VA et al. Chitosan but not chitin activates the inflammasome by a mechanism dependent upon phagocytosis. J. Biol. Chem. 286(41), 35447-35455 (2011).

18 Mori A, Oleszycka E, Sharp FA et al. The vaccine adjuvant alum inhibits IL-12 by promoting PI3 kinase signaling while chitosan does not inhibit IL-12 and enhances Th1 and Th17 responses. Eur. J. Immunol. 42(10), 2709-2719 (2012).

19 Guzman-Morales J, Ariganello MB, Hammami I, Thibault M, Jolicoeur M, Hoemann CD. Biodegradable chitosan particles induce chemokine release and negligible arginase-1 activity compared with IL-4 in murine bone marrow-derived macrophages. Biochem. Biophys. Res. Commun. 405(4), 538-544 (2011).

20 Hoemann CD, Guzman-Morales J, Tran-Khanh N, Lavallee G, Jolicoeur M, Lavertu M. Chitosan rate of uptake in HEK293 cells is influenced by soluble versus microparticle state and enhanced by serum-induced cell metabolism and lactate-based media acidification. Molecules 18(1), 1015-1035 (2013). 